\title{
TRAUMA OF THE LOWER URINARY TRACT
}

\author{
Anthony Deane
}

\section{Investigations in patients with lower urinary tract injuries}

- Inspect the urinary meatus for blood - Examine the abdomen for peritonism, perineal bruising, and a high riding prostate

- Perform intravenous urography to detect bladder perforation, displaced bladder, upper urinary tract injury

Perform cystography to exclude bladder perforation if patient has a catheter in place

Perform ascending urethrography (aqueous contrast) to exclude urethral injury (controversial)

\section{Bladder injuries}

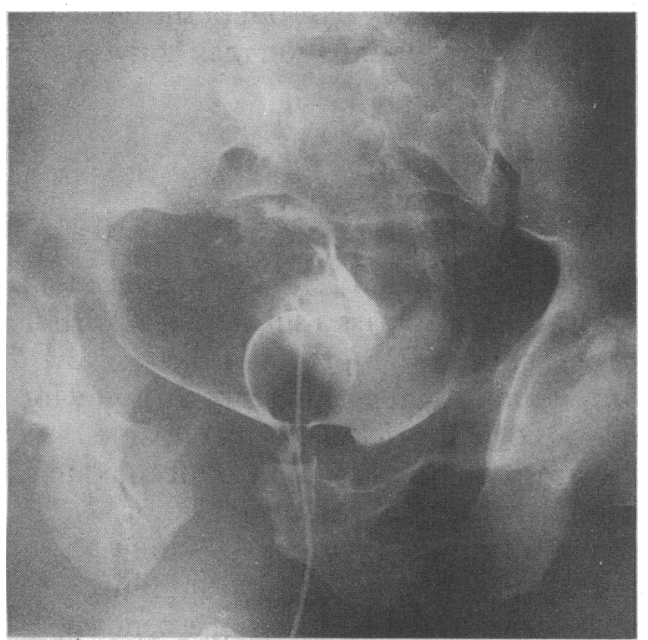

Cystogram of a bladder full of haematoma with extravasation of contrast due to a torn anterior bladder wall. The patient required 30 units of blood before bleeding was controlled by selective internal iliac embolisation.

\section{Bulbar injuries}

\section{Management of patients with bulbar injuries}

- Do not pass a urethral catheter

- If the patient passes urine give antibiotics and follow up

- If the patient has urinary retention insert a suprapubic catheter with a small calibre and give antibiotics. Perform urethrography after about five days and follow up
Injuries of the lower urinary tract cause more confusion than those of the upper urinary tract as their management is controversial. The rule in patients with suspected urethral injuries and major pelvic fractures is not to pass a urethral catheter without first seeking advice from a urologist. Even though monitoring urinary output is very important in patients with major injuries, a catheter should not be passed without careful thought. Urinary extravasation is not dangerous in the short term.

Bladder injuries may be associated with a pelvic fracture - that is, they may be caused by penetration by a bony fragment or by a direct blow to the lower abdomen, especially when the bladder is full. The condition may be missed in patients intoxicated with alcohol or those with a head injury. Patients with a bladder injury may have lower abdominal peritonism and not be able to pass urine. A catheter may have been passed by the receiving clinicians and the urine may contain blood.

Investigation begins by obtaining a plain radiograph to exclude pelvic fractures. Disruption of the pelvic symphysis alerts the clinician to the possibility of urethral injury and delayed rupture of the bladder due to stretching of the anterior bladder wall. An intravenous urogram may show an extravasation from a bladder injury. If there is no pelvic fracture and no urethral haemorrhage a urethral catheter may be passed, and cystography with $10 \%$ dilute contrast agent will show any important bladder injury. If an intraperitoneal bladder rupture is suspected then cystography should be done before diagnostic peritoneal lavage because a laparotomy will be required to repair such a lesion, if it is present, making lavage unnecessary. In patients with serious pelvic fracture, especially if it affects the pubic symphysis, upward dislocation of the bladder on urography should be excluded first, although this would usually be accompanied by urethral haemorrhage.

Treatment-Patients with important intraperitoneal ruptures with peritonism are best treated by laparotomy and drainage by suprapubic catheter as well as by urethral catheter for about seven days. Broad range antibiotics should be given. Extraperitoneal injuries are managed by drainage by catheter without irrigation for about 10 days. A catheter of least $20 \mathrm{FG}$ is necessary, and cystography to confirm healing is advisable before withdrawal of the catheter.

Bulbar injuries occur by direct trauma - for example by falling on to a bicycle crossbar. Occasionally thay can be caused by penetrating trauma. Patients with bulbar injuries have perineal bruising and blood at the urinary meatus. A urethral catheter must not be passed as it may aggravate the injury and introduce infection. The patient should be treated expectantly, and, if he or she passes urine, should be given antibiotics and followed up. Patients with urinary retention should be treated by inserting a suprapubic catheter with a small calibre percutaneously as heavy haematuria is not usually a problem. Antibiotics are given, and urethrography can be performed after about five days. The patient will need urological follow up to exclude formation of stricture. 


\section{Urethral injuries caused by pelvic fracture}

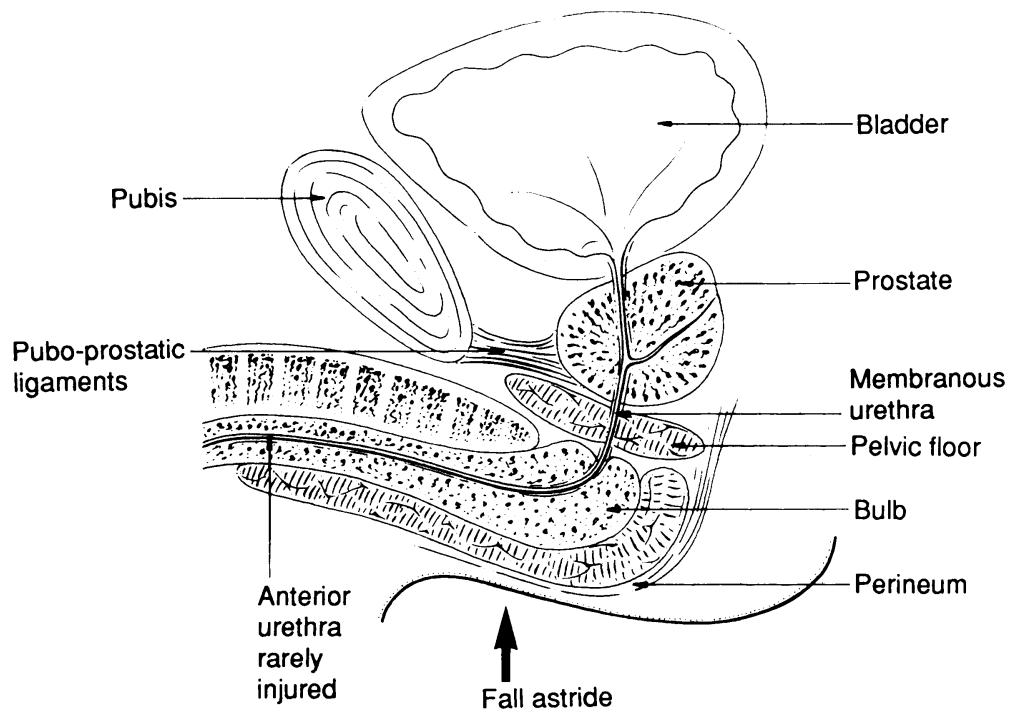

The membranous urethra below the prostate is damaged in about $10 \%$ of men with pelvic fractures. Serious injuries, though rare, are devastating as impotence and stricture are common sequels. Damage usually comprises a partial tear, but, occasionally, complete disruption and upward dislocation of the bladder and prostate occurs. The prostate is fixed to the pubic symphysis by the puboprostatic ligaments, and any severe disruption of the pubic symphysis is liable to tear the prostate off the membranous urethra, which is attached to the pelvic floor.

The puboprostatic ligaments carry the prostate with them in patients with pelvic fracture, tearing the urethra off the pelvic floor.

\section{Signs of membranous urethral injury}

- Pelvic fracture

- Perineal bruising

- Blood at the meatus

- Inability to pass urine

- High riding prostate
The signs of urethral injury caused by pelvic fracture are blood at the meatus, perineal bruising, and inability to pass urine. A urethral catheter must not be passed as it may aggravate the injury, even if it passes the tear: the urethra is often traumatised and devascularised and may be eroded by the catheter or disintegrate around it; the catheter will prevent the haematoma from draining and introduce infection with possible fistulation on its withdrawal; and worst of all, the catheter may pass out of the tear and drain blood and urine below the prostate. Balloon inflation may convert a partial disruption into a complete one.

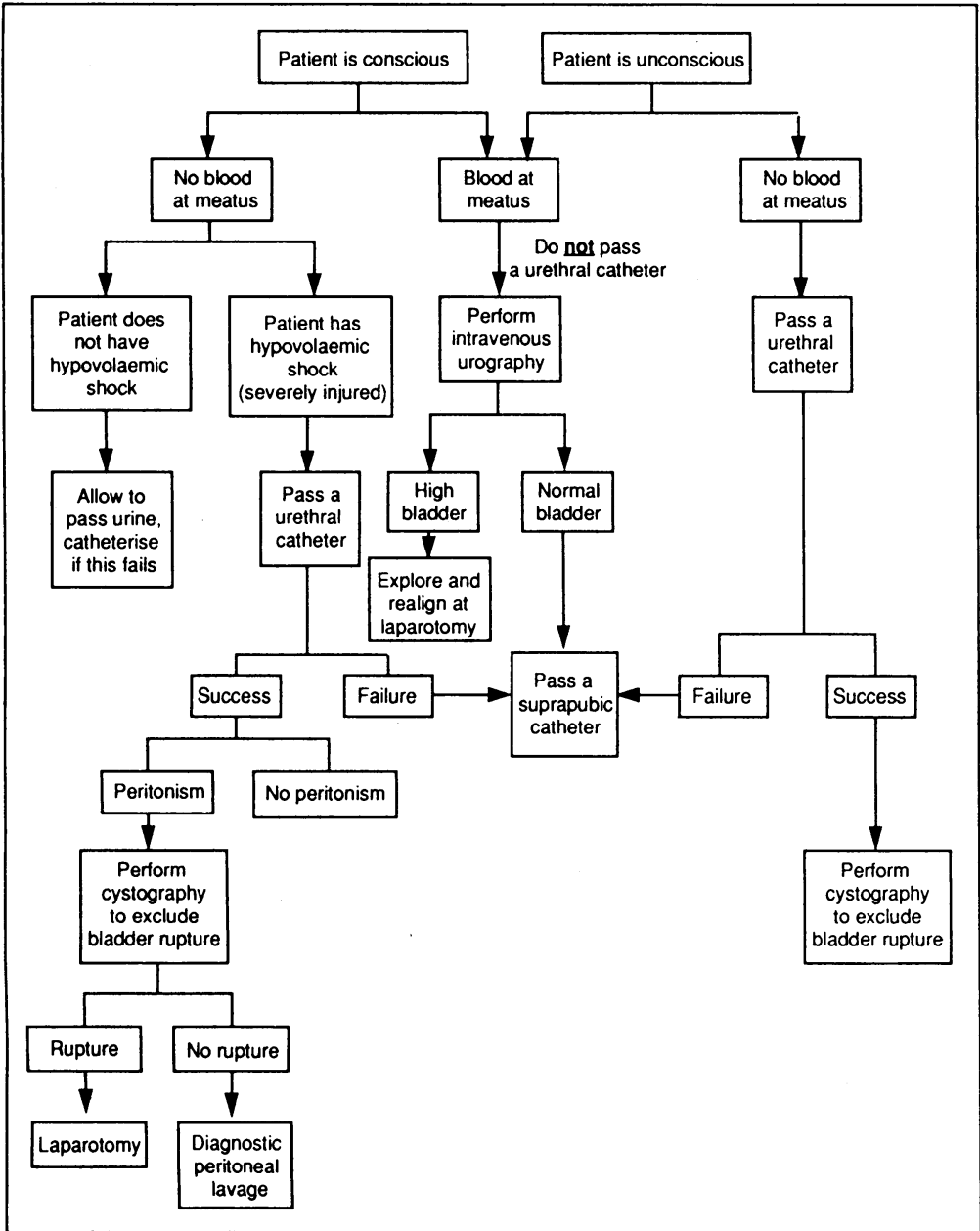

The safest way to treat urethral injuries caused by pelvic fracture is to pass a suprapubic catheter of adequate calibre either percutaneously or by cystotomy if the patient requires a laparotomy for other reasons or if the bladder is impalpable. Intravenous urography should be performed to exclude total disruption with a high riding bladder above the pubic symphysis - an indication for exploration and repositioning. Some authorities advise performing ascending urethrography to delineate the extent of the injury and plan its management, but this can be difficult in the emergency room. In general a urethral catheter can be passed in a patient with a pelvic fracture if there is no blood at the meatus or if the pubic symphysis is not severely disrupted on radiography. If any difficulties are encountered urological help should be summoned and an ascending urethrogram considered or suprapubic catheteristation performed. Later treatment of these injuries entails urethroscopy, but late strictures are very common.

In women the urethra is injured only rarely, and usually a catheter can easily be passed in those with pelvic fractures and, if necessary, cystography performed to exclude the possibility of bladder injuries.

Urological management of men with serious pelvic fractures. 


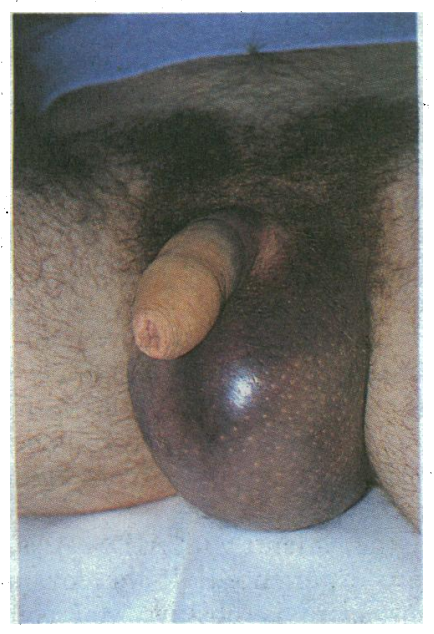

Scrotal haematoma.
Serious injuries to the penis and scrotum are unusual. The mobile scrotal skin can be used to cover penile defects and has good powers of recovery. Scrotal tears heal well without suturing. The erectile mechanism should always be repaired if torn with monofilament non-absorbable sutures.

The testicles can be damaged by direct trauma - usually a blow or a kick. If bleeding is confined to the scrotal skin no active treatment is required. Tense haematoceles should, however, always be explored as these usually indicate that the testis is torn and needs repair. Severe damage may require orchidectomy.

The picture of scrotal haematoma was provided by Mr Andrew Cope, St Bartholomew's Hospital.

Mr Anthony Deane, FRCs, is consultant urologist, William Harvey Hospital, Dover, Kent, and Buckland Hospital, Ashford, Kent.

The ABC of Major Trauma has been edited by Mr David Skinner, FRCs; Mr Peter Driscoll, FRCS; and Mr Richard Earlam, FRCS.

\title{
Letter from . . . Chicago
}

\section{Rounds}

\author{
George Dunea
}

From time to time on rounds I discuss with the new students and residents the principles of history taking. I talk about the great Sir William Osler, hero of all bedside pedagogues, who urged his students to "listen to the patient, he is telling you the diagnosis." I mention Sir Thomas Lewis, also great though lacking name recognition nowadays, who expected his interns to know how to examine a patient but not as yet to have enough experience to obtain a good history. Then I refer to the late Michael Balint, the psychiatrist, who wrote, "If you ask questions you will get answers but precious little else."

Next I try to bridge the gap between theory and practice, for instructors must be men of the world to command the residents' respect. I concede that life is too short for mere listening and grunting encouragingly-except for the psychoanalysts. Betraying my age, I refer to Paul Woods's introduction to his 1955 book, in which he recommends the skilful use of leading questions, such as "Does the pain tingle?" I also mention Sam Levine's technique of asking the opposite of what he expected to hear-such as "Is your chest pain worse when you rest?" or "Is your joint stiffness worse in the evening?" Ideally, I say, there should be only one history, not seven specialists' subjective perspectives like in the Japanese tale of Rashomon.

Drawing from Alvan Feinstein's observations but leaving out the Greek, I make a distinction between the main problem and what took the patient to the doctor-what he called the iatropic stimulus. In the manner of Gibbon I suggest that the failure of the once popular problem oriented record might be due not to who gave it but to whom it was given. I ask instead that progress notes be short and to the point, emphasising assessment of the clinical state and plans for the future. I warn against trying to write down the history in its final form while taking it to avoid breaking the flow with interruptions. I advise the students to construct complex histories not so much along symptoms as along the sign posts that patients are likely to remember: being admitted to a hospital bed, cut by a surgeon, or seen in a doctor's office. I talk about the need to sometimes go into great detail, referring to the once obligatory exercise of writing 15 page case histories and I quote Voltaire's dictum about doctors giving medicines they know nothing about to patients they know nothing about. At this juncture I stop short of items such as empathy, sympathy, putting yourself in the patient's shoes - which is just as well because the students are getting restless and anxious to get to the noon lecture on the prostaglandin cascade.

We meet again at $2 \mathrm{pm}$ for rounds. The intern is puzzled at my insistence on hearing the history at the bedside. Their previous attending physician, he says, first wrote his notes in the conference room while the interns presented their findings - a compromise, I reflect, between Feinstein's abstract and veterinary forms of rounds. The first patient is an anxious 66 year old woman with oxygen prongs up her nose, being "stepped down" after three days of ruling out myocardial infarction in the intensive care unit. She is "an unstable angina," explains the intern; she also has hypertension and congestive heart failure.

When I ask how long she has had hypertension he turns to the woman and asks "How long have you had high blood pressure?" When I ask about previous hospital admissions he says that she has had a "cardiac cath" and that he will send off for the records. Trying to practise what was preached in the morning, we learn that she spent two weeks in intensive care 20 years ago for an alleged heart attack after her dentist discovered high blood pressure. Suspicious and somewhat peeved at first at being asked so many details, she remembers having had a cardiac catheterisation in 1980 and being told she had a funny valve. Chest pains began after a car accident in 1984, usually starting in the middle of the back and radiating round both sides to the front. Her right shoulder also hurts. It hurts to turn over in bed, she says. 